\title{
ABCD1 GENE MUTATIONS IN CHINESE PATIENTS WITH ALD
}

Thirty-two different $A B C D I$ mutations were identified by direct sequencing of polymerase chain reaction products in 34 unrelated Chinese X-linked adrenoleukodystrophy (ALD) patients examined at Peking University First Hospital, Beijing, PRC. Eight of 10 screened sisters and cousins were carriers. Mutational heterogeneity is prevalent in Chinese patients with ALD, a finding consistent with other populations. Mutational analysis of ABCVD1 gene is of value in counseling of ALD families. (Pan $\mathrm{H}$, Xiong $\mathrm{H}, \mathrm{Wu}$ Y et al. ABCD1 gene mutations in Chinese patients with X-linked adrenoleukodystrophy. Pediatr Neurol August 2005;33:114-120). (Respond: Dr Xi-Ru Wu, Department of Pediatrics, Peking University First Hospital, No 1 of Xi An Men Street, Beijing, People's Republic of China).

COMMENT. Direct sequencing of polymerase chain reaction products is a simple and efficient method for diagnostic mutational analysis of the $\mathrm{ABCD} 1$ gene found in the majority of X-linked ALD patients. X-linked ALD is transmitted maternally, and analysis of the ABCD1 gene in women with an affected family member can determine the risk of transmission to offspring, of help in genetic counseling.

\section{LORENZO'S OIL IN TREATMENT OF ASYMPTOMATIC ALD}

The effect of 4:1 glyceryl trioleate-glyceryl trierucate (Lorenzo's oil) on disease progression in 89 asymptomatic boys (mean age $4.7+/-4.1$ years; range $0.2-15$ years) with Xlinked adrenoleukodystrophy (ALD) and normal brain MRI was studied at Johns Hopkins University, Baltimore, MD, and other centers. Therapy was initiated before 7 years of age in 64 patients. Sixty-six patients $(74 \%)$ had no neurologic deterioration at a mean follow-up of 6.9 years, 21 (24\%) developed MRI abnormalities, and $8(9 \%)$ had both neurologic and MRI abnormalities. Those with neurologic abnormalities had the childhood cerebral phenotype. Boys with an elevated plasma hexacosanoic acid had a significantly increased risk of more rapid progression and MRI abnormalities. Lowering of the hexacosanoic acid plasma level following treatment with Lorenzo's oil (LO) was associated with a reduced risk of developing MRI abnormalities. LO therapy is recommended in asymptomatic patients with X-linked ALD and normal MRI. (Moser HW, Raymond GV, Lu S-E et al. Follow-up of 89 asymptomatic patients with adrenoleukodystrophy treated with Lorenzo's oil. Arch Neurol July 2005;62:1073-1080). (Respond: Hugo W Moser MD, Kennedy Krieger Institute, 707 N Broadway, Baltimore, MD 21205).

COMMENT. Lorenzo's oil therapy is recommended in boys, especially those $<7$ years of age, with ALD who are asymptomatic, have normal MRI, and are at risk of developing the childhood cerebral form of ALD. The authors stress that this recommendation is based on suggestive, not definitive, evidence of a preventive effect. Potential patients are identified by assays of very long-chain fatty acids in at-risk relatives of patients with known ALD or idiopathic Addison's disease. 